\title{
Hypersonic shock wave evidence in RR Lyrae stars ${ }^{\star} \star \star \star$ First detection of neutral line disappearance in S Arae
}

\author{
M. Chadid ${ }^{1,2}$, J. Vernin ${ }^{1,2}$, and D. Gillet ${ }^{3}$ \\ 1 Observatoire de la Côte d'Azur, Université de Nice Sophia-Antipolis, UMR 6525, Parc Valrose, 06108 Nice Cedex 02, France \\ e-mail: chadid@unice.fr \\ 2 Antartic Research Station, Dome C, TAAF, Antarctica \\ ${ }^{3}$ Observatoire de Haute Provence, CNRS, 0478 Saint Michel l'Observatoire, France
}

Received 27 May 2008 / Accepted 30 July 2008

\section{ABSTRACT}

\begin{abstract}
Context. We carried out a new high resolution spectroscopic analysis of S Arae, one of the largest amplitude RR Lyrae star, to constrain the dynamical structure of the outermost layers of RR Lyrae stars.

Aims. New high precision spectroscopic observations of S Arae were obtained using UVES at VLT-UT2 and FEROS at ESO-1.52 telescopes, during the rise of the S Arae light curve, over an entire pulsation period and covering a large wavelength range, from 376 to $1000 \mathrm{~nm}$.

Methods. We used the first line moment method to measure the radial velocity and to establish the radial velocity curves. The atmosphere stratification was done by the line identification method derived from the Kurucz atmosphere models.

Results. For the first time, we show the existence of hypersonic shock waves in the photosphere of RR Lyrae stars. Our spectroscopic results reveal a new mechanism: the neutral line disappearance phenomenon. All neutral metallic absorption lines, over the whole spectrum, undergo a total line disappearance during the hump, while the ionized lines show line doubling connected by the two-step Schwarzschild's mechanism. Thus, a hypersonic shock wave propagating through the photosphere around the maximum light phase results in the line disappearance. The shock energy is high enough to ionize the atoms, forming the layer crossed by the shock wave. The shock at first recedes, then becomes stationary and finally advances with a very high Mach number crossing the high atmosphere of the star. We stress the need for additional theoretical efforts to come to a better understanding of hypersonic shock wave and dynamic pulsational motion relationship.
\end{abstract}

Key words. shock waves - hydrodynamics - stellar dynamics - techniques: spectroscopic - techniques: radial velocities stars: variables: RR Lyr

\section{Introduction}

Since the spectroscopic works of Preston (1961) and Oke (1966) regarding one of the largest amplitude RR Lyrae stars: X Ari, only a small number of studies have been devoted to these kinds of stars. Indeed, high resolution spectroscopy requires the largest available telescopes especially when rapid phenomena occur within the stellar atmosphere. Contrary to classical Cepheids, the atmospheric dynamics in RR Lyrae stars are relatively well studied only in the brightest RR Lyrae: RR Lyr. Consequently, our knowledge of the atmospheric behaviour of RR Lyrae stars is still limited.

As mentioned by Willson \& Hill (1979) and Bowen (1988), the shock waves are certainly at the origin of the mass loss and of the formation of circumstellar envelopes. Iroshnikov (1962) interpreted the observations of metallic and hydrogen line radial velocities, splitting of the spectral lines and hydrogen emission lines of RR Lyr made by Sanford (1949) with the help of a model in which the shock wave propagates through an exothermic gas in the atmosphere of RR Lyr. He concluded that the discontinuity

\footnotetext{
* Based on observations obtained at the European Southern Observatory in Chile in the programs P67.D-0321(B) and P67.D-0321(A).

$\star \star$ Tables 1 and 2 are only available in electronic form at

http://www . aanda.org
}

in the radial velocity curve, the emission and double absorption hydrogen lines, at the maximum of the light curve, would be the consequence of shock waves propagating through the atmosphere of RR Lyr.

Using a nonlinear nonadiabatic pulsational hydrodynamic model, Hill (1972) interpreted the presence of a hump and a bump in the RR lyr light curve as a consequence of a strong shock wave, "the main shock" produced by the opacity mechanism giving the pulsation and a secondary shock "the early shock" produced by the colliding of the upper atmospheric layers with deeper ones during the infall phase. Using high spectral resolution $(0.2 \AA)$ observations of the $\mathrm{H} \alpha$ profile in X Ari, Gillet \& Crowe (1988) detected a weak blue emission during the bump while during the hump, another stronger blue emission is observed, followed by the doubling phenomenon. They associated the hydrogen emission and doubling phenomena with the propagation of the main shock and early shock in X Ari. Their results are consistent with Hill (1972). Gillet and Crowe claimed to have detected a very weak red emission during the hump. Nevertheless, neither observations nor theoretical studies confirmed this effect.

The first and only detection of the doubling phenomenon over metallic absorption lines in RR Lyrae stars was reported by Chadid \& Gillet (1996a). A resolving power of 42000 and a time resolution around $1 \%$ of the pulsation period of RR Lyr 
were necessary. They interpreted the phenomenon as the consequence of a "two-step" Schwarzchild mechanism (1952). The shock is at first receding for the observer, becomes stationary and then advances. These three phases of the shock evolution are confirmed using a nonlinear nonadiabatic radiative hydrodynamic model and Van Hoof effect theoretical studies (Chadid et al. 2000). However, up to now, none of the RR Lyrae theoretical models are able to resolve the fine shock structure. The spatial resolution near the shock front is too weak to resolve even the largest wake zone where the photo-recombinations occur (see Bono et al. 1994, 1997; Fokin et al. 2000; Gillet et al. 1989; Chadid \& Gillet 1996a). This means that they cannot correctly give the post shock and the shock front velocities. However, they correctly predict the amplitude of the shock, i.e. the difference between hydrodynamical velocities "before" and "behind" the shock wave.

In order to evaluate new studies of the atmospheric dynamics in RR Lyrae, especially in the largest amplitude RR Lyrae stars and to establish new observational signatures of the shock wave, we obtained a new series of high resolution spectroscopic observations, with a wide spectral coverage of S Arae.

\section{The data}

\subsection{High resolution spectroscopic observations}

Using the high resolution UV-Visual Echelle Spectrograph UVES (Kaufer et al. 2004) at the VLT-UT2 (Kueyen) on Cerro Paranal, we carried out, on July 23rd, 2001, spectroscopic observations of S Arae, one of the largest amplitude RR Lyrae stars, whose pulsation period is 0.452 days and $V$-magnitude is between 10 and 11.5. Thirty five measurements were done, during the rise of the light curve $-40 \%$ of the pulsation period - using the dichroic \#2 and the standard setting centred at $437 \mathrm{~nm}$ in the blue arm, covering the 376-500 range and $860 \mathrm{~nm}$ in the red arm, covering the 672-1000 range. The exposure time was $240 \mathrm{~s}$ for both arms, giving an average time resolution of $0.5 \%$. The detector in the blue arm is an EEV CCD, while in the red it is a mosaic of one EEV and one MIT CCD. All CCDs are composed of $4096 \times 2048$ square pixels of $15 \mu$ side. The slit width was $0 .{ }^{\prime} 8$ in both arms. The final combined spectrum has been rebinned to a constant pixel size of $0.005 \mathrm{~nm}$ and covers the wavelength range $376-1000 \mathrm{~nm}$. The average resolving power $R$ is around 45000 for both arms and was measured from the FWHM of the thorium lines of calibration spectra. The signal-to-noise ratio of the final spectrum is about 80 per resolution element at $400 \mathrm{~nm}$, 120 at $500 \mathrm{~nm}, 110$ at $600 \mathrm{~nm}$ and 70 at $800 \mathrm{~nm}$.

In order to achieve an extended coverage in the pulsation period, we observed S Arae continuously during one pulsation period, using the FEROS instrument (Pritchard 2004) at the ESO $1.5 \mathrm{~m}$ telescope on Cerro La Silla. Twenty seven measurements were performed for $12 \mathrm{~h}$ on June 29-30th, 2001. The spectral resolution is 48000 , the spectral coverage $370-860 \mathrm{~nm}$ and for an exposure time of $900 \mathrm{~s}$, an average time resolution of $2.5 \%$, and an average signal-to-noise ratio of 50 .

The phasing of the observations was adopted using the ephemeris:

$\operatorname{HJD}($ max.light $)=2452137.556+0.451883 \mathrm{E}$.

The maximum of the light and the period are given by A. Paschke (2001, private communication).

\subsection{Data reduction}

Both UVES and FEROS data were reduced using the ECHELLE context of MIDAS and each CCD was treated independently. The reduction included background subtraction, cosmic ray filtering, flat fielding, extraction, wavelength calibration and order merging. We have normalized each spectrum to its continuum with an accuracy of around $1 \%$ using a polynomial formula. The data were corrected for earth motion and we brought the wavelength scale back to the stellar restframe of S Arae assuming a star velocity $V_{*}$ with respect to the solar system barycenter of $183.31 \mathrm{~km} \mathrm{~s}^{-1}$ (see Sect. 3).

To confirm the estimated error of the radial velocity measurements of both UVES and FEROS, we measured the radial velocity of the standard star HD 182572, $V$-magnitude 5, G8IV spectral type and $-100.10 \mathrm{~km} \mathrm{~s}^{-1}$ radial velocity, according to Perryman et al. (1997) at the beginning of both UVES and FEROS observing nights. Data calibration was performed in the same manner as the $\mathrm{S}$ Arae spectra. The $\mathrm{S} / \mathrm{N}$ ratio in the HD 182572 spectra was around 200 and 90 for UVES and FEROS.

\subsection{Line identification and radial velocity measurements}

The radial velocity measurements were obtained with the first line moment method. According to Chadid \& Gillet (1996a,b), the differences between this method and the simple Gaussian fit occur only when the line doubling phenomenon appears. The radial velocity measurements of the standard star HD 182572 were obtained in the same manner and showed uncertainties of around $100 \mathrm{~m} \mathrm{~s}^{-1}$ and $500 \mathrm{~m} \mathrm{~s}^{-1}$ respectively for UVES and FEROS radial velocity measurements. The line identification was derived in a consistent way using Kurucz atmosphere models (CD-ROOM 23, Kurucz 1993) and were computed for the best estimated of S Arae parameters.

\section{Photospheric motion parameters}

Figure 1 shows UVES and FEROS heliocentric radial velocities of a set of five single ionized metallic absorption iron lines, in the blue and visible spectrum, between $370-570 \mathrm{~nm}$, respectively during the ascending branch of the light curve (between 0.82-1.086 pulsation phases) and one complete pulsation period. As one can see, there are significant differences in the descending branch between FEROS radial velocity curves and those of UVES. Two explanations will be discussed in detail in the next paper (in preparation). First, we suspect the multiperiodic character of the S Arae pulsation, the Blazhko effect (Blazhko 1907), as suggested recently by Wils \& Sodor (2005). The second explanation is consistent with previous results of Chadid (2000), who pointed out that important irregularities in the RR Lyrae atmosphere occur during successive pulsation cycles, clearly showing significant differences in radial velocity curves from one pulsation cycle to the next. One of Chadid's plausible explanations of the irregularities is the nonlinear dynamical interaction between the motion of the highest and lowest metallic layers, when strong outward shock waves traverse them.

The FEROS radial velocity curves of the single ionized metallic absorption iron lines show a very small elbow around phase 0.71 . This phenomenon had already been observed in a few bright RR Lyrae stars such as X Ari (Jones et al. 1987) and RR Lyr (Chadid \& Gillet 1996a) and is connected to the bump in the light curve (see Fig. 2). The descending branch of the radial velocity curve occurs during a very small phase interval, 


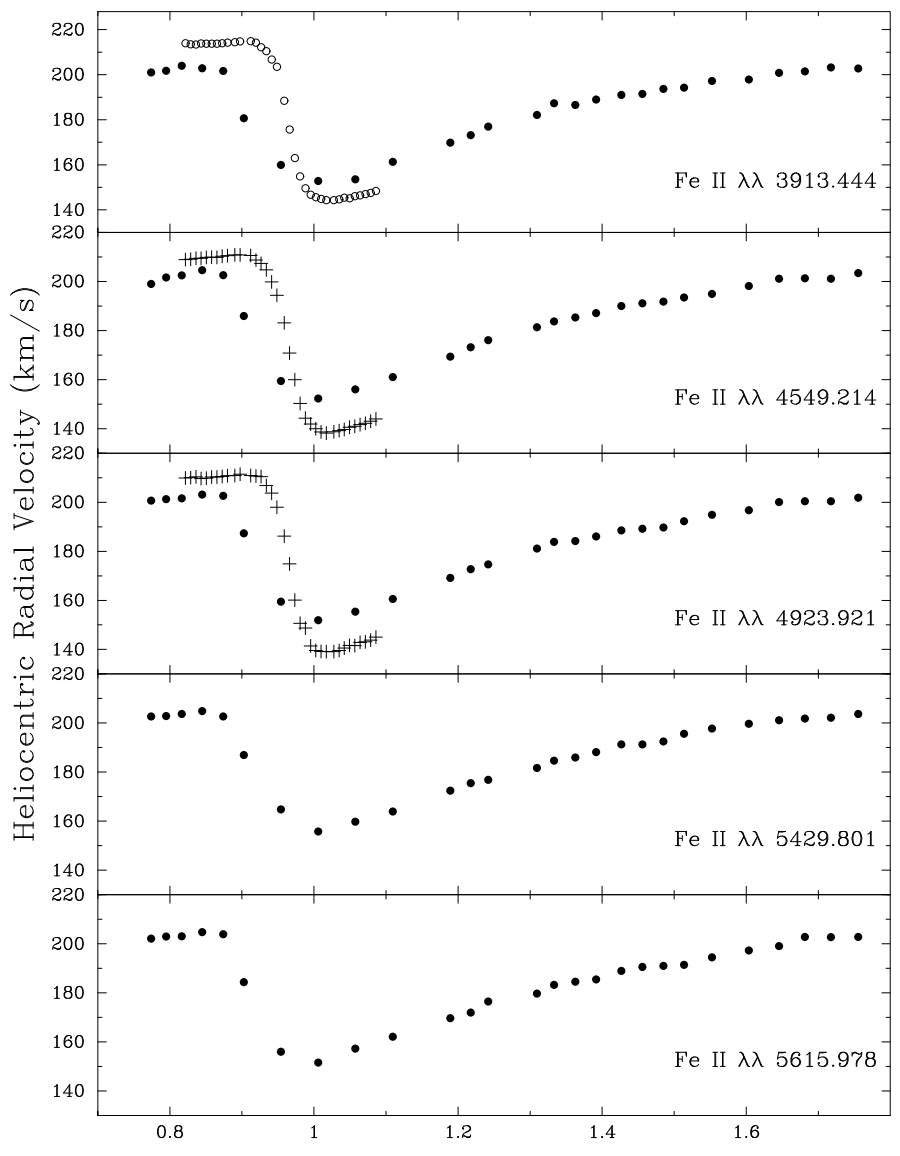

Pulsation phase

Fig. 1. UVES and FEROS heliocentric radial velocity curves (plus and filled circles respectively) for some single ionized metallic absorption iron lines.

the so-called rising time, corresponding to $\sim 10 \%$ of the pulsation period. Such a small rising time has never been observed in RR Lyrae stars. We recall a rising time of $\sim 19 \%$ of the pulsation period in RR Lyr (Chadid \& Gillet 1996a) and 14\% in X Ari (Jones 1987).

From the FEROS heliocentric radial velocity $V_{\mathrm{r}}(t)$, we can derive the pulsational velocity $\dot{R}(t)$ in the stellar rest frame using:

$\dot{R}(t)=-\beta\left(V_{\mathrm{r}}(t)-V_{*}\right)$

where $\beta$ is the value of the correction factor for geometrical projection and limb darkening and $V_{*}$ is the center-of-mass velocity. Since the ionized metallic absorption iron lines Fe II are formed near the photosphere, $\dot{R}(t)$ is close to the photosphere motion and $V_{*}$ is assumed to be equal to the so-called $\gamma$-velocity, i.e., the average value of the heliocentric radial velocity curve over one pulsation period. Here, we adopt for $\beta$ a value of 1.36 as discussed by Burki (1982). Using a Fourier series fit, the derivative of $\dot{R}(t)$ gives the acceleration $\ddot{R}(t)$ of the stellar surface which refers to the formation region of single ionized iron lines Fe II $\lambda \lambda 3913.444,4549.214,4923.921,5429.801$ and 5615.978, as shown in Fig. 3. An integration of $\dot{R}(t)$ gives the radius variation:

$R(t)-R_{0}=\Delta R(t)=\int \dot{R}(t) \mathrm{d} t$

where $R_{0}$ is the mean stellar radius. Figure 4 shows the radius variation of the five ionized iron stellar regions. The average

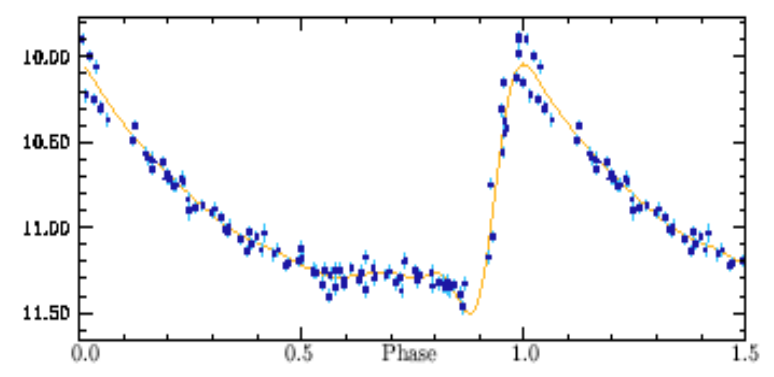

Fig. 2. S Arae $V$-light curve from the Hipparcos satellite. The hump is not clearly marked and the bump is relatively flat and centred at phase 0.7 .

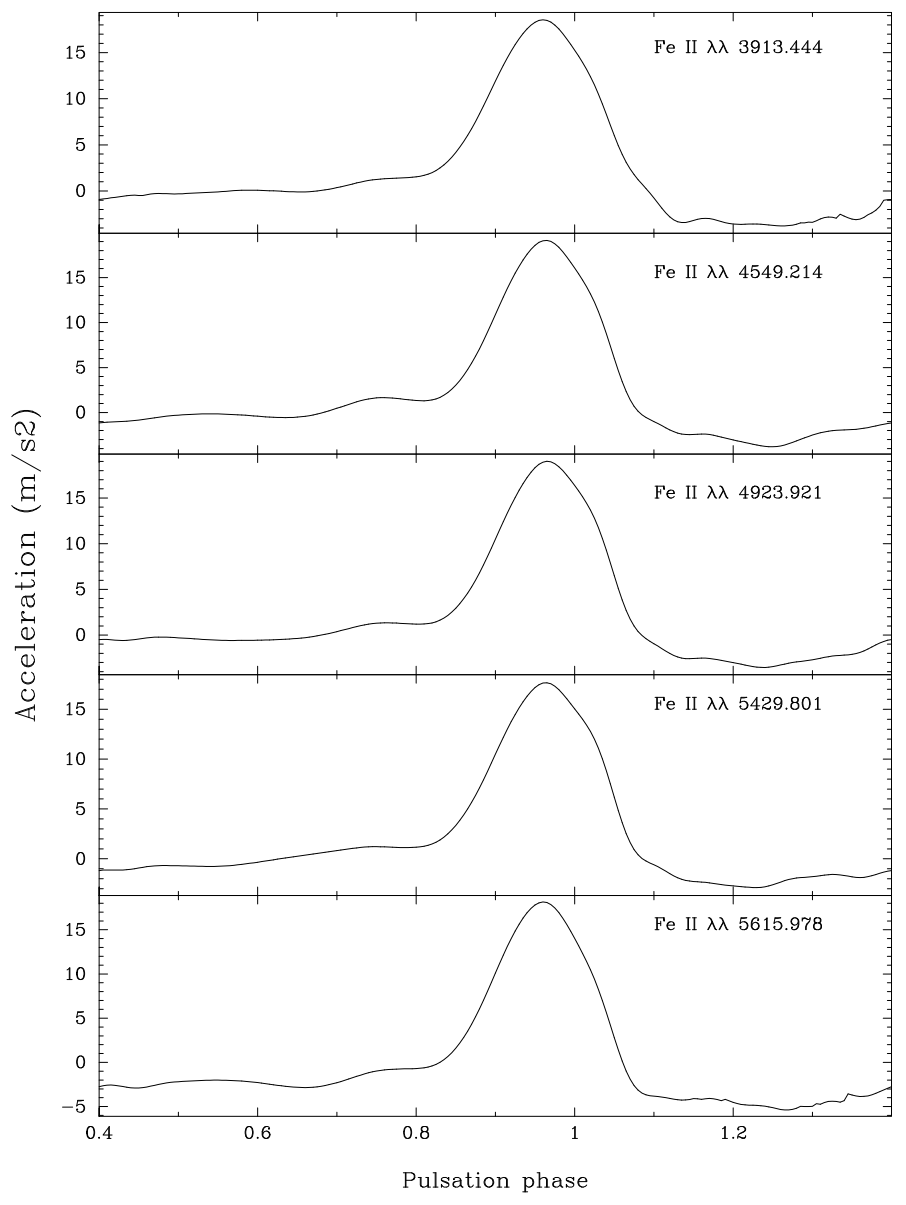

Fig. 3. Calculated curve of acceleration $\ddot{R}(t)$ for some single ionized metallic absorption iron lines.

value of the heliocentric radial velocity curve over one pulsation period for five ionized iron lines is given in Table 3. We determine a center-of-mass velocity value of $183.31 \mathrm{~km} \mathrm{~s}^{-1}$. The acceleration curves, shown in Fig. 3, are characterized by a double acceleration peak. The main acceleration peak occurs during the hump observed in the luminosity curve around the phase 0.92 (Fig. 2), corresponding to the minimum radius phase (Fig. 3). The maximum average value of the main acceleration is around $1930 \mathrm{~cm} \mathrm{~s}^{-2}$ while the secondary acceleration is $90 \mathrm{~cm} \mathrm{~s}^{-2}$ and corresponds to the elbow of the radial velocity curve around the phase 0.71 . The secondary acceleration is 21 times smaller than the main one. 


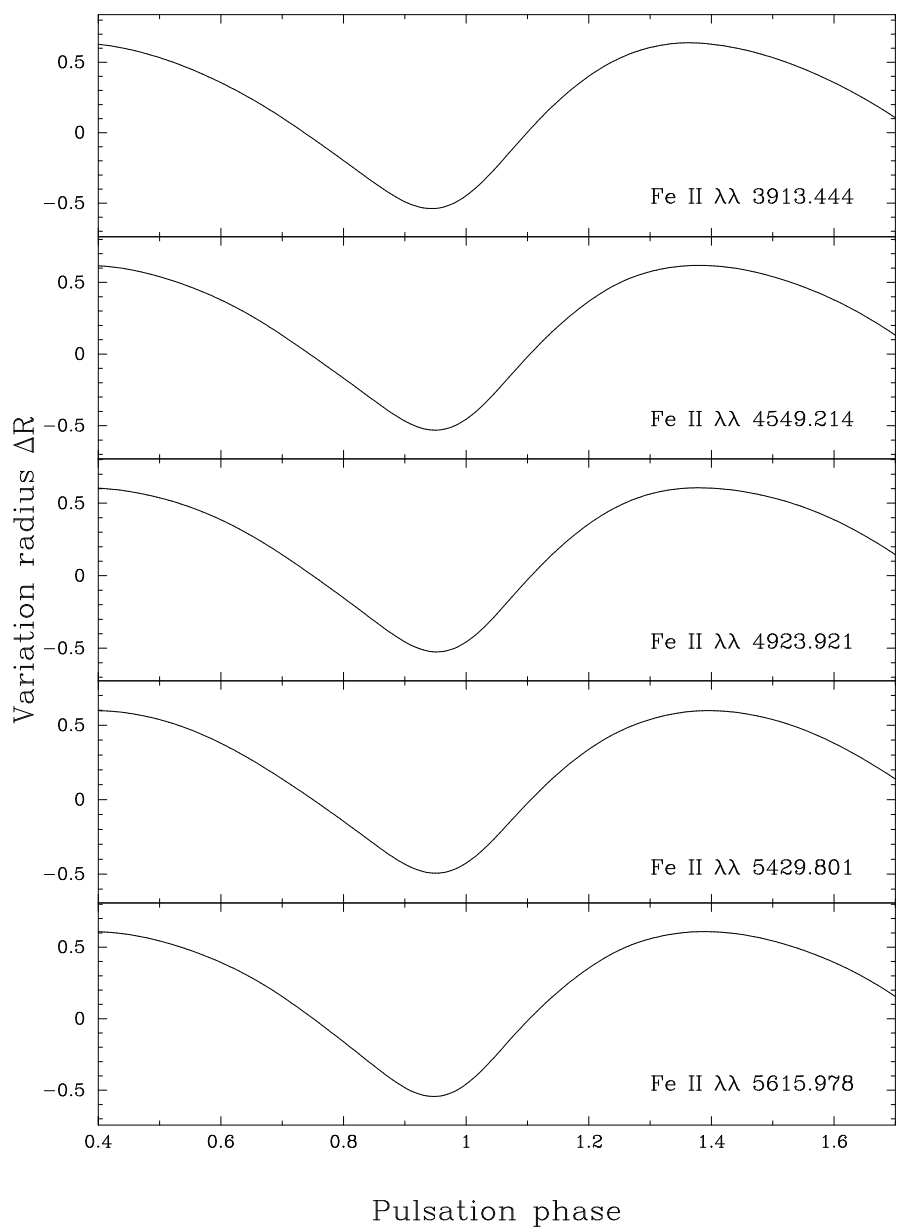

Fig. 4. Calculated curve of radius variation $\Delta R(t)$ for some single ionized metallic absorption iron lines.

The average star gravity is given by the so-called Newton gravity

$g_{*}=G \frac{M}{R^{2}}$

where $G$ is Newton's gravity constant $\left(G=6.67 \times 10^{-11}\right), M$ and $R$ are respectively the mass and radius of the star. Assuming an $\mathrm{S}$ Arae mass of $0.65 M_{\odot}$ and an S Arae photospheric radius of $4.7 R_{\odot}$, we calculated an average stellar gravity of $801 \mathrm{~cm} \mathrm{~s}^{-2}$. We conclude that the dynamical gravity given by the peak of the main acceleration in S Arae is 2.5 time larger than the stellar gravity $g_{*}$. Thus the intensity of the pulsating motion in S Arae is stronger than the one occurring in RR Lyr. A ratio around 1.8 was given by Chadid \& Gillet (1996a). In Classical Cepheid stars, this ratio was estimated to lie between $1(\delta$ Cep $)$ and 1.8 (X Cyg).

Table 3 gives the relative radius variation for the five iron layers. We determine a main relative radius variation $\frac{\Delta R_{\mathrm{ph}}}{R_{\mathrm{ph}}}$ around $24.33 \%$. Chadid \& Gillet (1996a) found $14.60 \%$ for RR Lyr.

All these results show that the RR Lyrae stars have an extended atmospheric motion slightly larger than those of Classical Cepheids which are reported to have a value of $22 \%$ (Breitfellner \& Gillet 1993).
Table 3. Main dynamic parameters of different $\lambda \lambda$ Fe II absorption lines. The first column gives the wavelength, the second the average radial velocity over one pulsation period, the third the primary acceleration, the fourth the secondary acceleration and the fifth the relative radius variation.

\begin{tabular}{lcclc}
\hline \hline$\lambda \lambda$ Fe II & $\begin{array}{c}\gamma \text {-Velocity } \\
\left(\mathrm{km} \mathrm{s}^{-1}\right)\end{array}$ & $\begin{array}{c}\text { Primary acc. } \\
\left(\mathrm{cm} \mathrm{s}^{-2}\right)\end{array}$ & $\begin{array}{l}\text { Secondary acc. } \\
\left(\mathrm{cm} \mathrm{s}^{-2}\right)\end{array}$ & $\begin{array}{c}\Delta R / R_{\mathrm{ph}} \\
(\%)\end{array}$ \\
\hline & & & & \\
3913.444 & 183.74 & 1925.56 & 84.70 & 24.69 \\
4549.214 & 183.45 & 1820.77 & 91.88 & 23.56 \\
4923.921 & 182.85 & 1981.35 & 75.22 & 24.42 \\
5429.801 & 183.38 & 1820.07 & 91.88 & 23.96 \\
5615.978 & 183.10 & 2100.45 & 113.93 & 25.01 \\
\hline
\end{tabular}

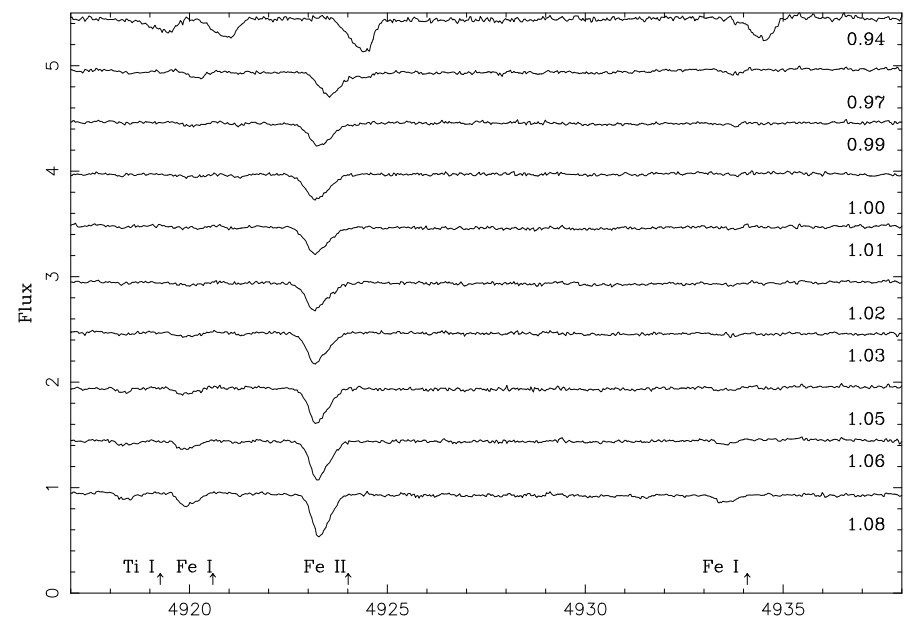

Stellar rest frame wavelenght $(\AA)$

Fig. 5. UVES observed profiles of neutral and single ionized metallic absorption lines associated with the hump. The neutral line shows line disappearance. The phase is labeled in the lower right corner. The small vertical lines give the position of the laboratory line wavelength.

\section{Neutral line disappearance phenomenon}

Figures 5 and 6 show respectively the UVES and FEROS observed line profile evolution of neutral and single ionized metallic absorption lines during the hump phases. The most striking feature is the disappearance of the neutral metallic absorption lines during the phase interval $0.97-1.05$, i.e., around the luminosity maximum, while the ionized lines only undergo a decrease of the equivalent width. The line disappearance phenomenon occurs for $8 \%$ of the pulsation period and affects all neutral metallic absorption lines, such as Fe I, Mg I, Ti I and Ca I detected over the observed spectral range from $370-570 \mathrm{~nm}$ in both FEROS and UVES data.

For the first time, the neutral line disappearance in pulsating atmospheres is reported here. This phenomenon is strongly connected to the main shock passage across the atmosphere of S Arae. Indeed, during the hump, around luminosity maximum, the main shock intensity is largest where its energy is transformed into thermal energy. The electron temperature increases more to reach the temperature of the first ionization level of heavy particles (atoms within the layer crossed by the shock). The energy of the main shock is sufficient to ionize the metallic neutral elements but not large enough to ionize the metallic ionized elements. No ionized line disappearance phenomenon is observed. 


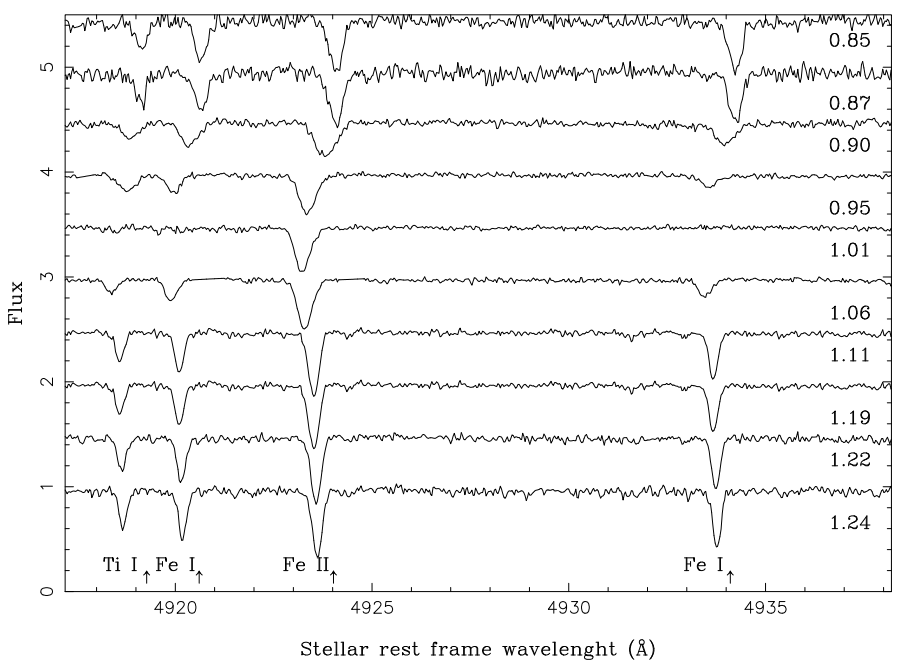

Fig. 6. As for Fig. 5 with the FEROS observed profile.

\section{Line doubling phenomenon}

Figure 7 shows the UVES observed profiles of the single ionized metallic absorption line Fe II $\lambda \lambda 4923.921$ associated with the hump. The exposure time of $4 \mathrm{mn}$, and the time resolution of $0.5 \%$, is short enough to give small details at each step of the evolution of the doubling mechanism. A line doubling phenomenon is well observed at phase interval 0.927-0.974. First, two redshifted components appear, the left one being weaker than the red one. At phase 0.949 , a flux equality of the two line components is clearly observed with a residual flux around 0.7 and the left component is centred around the laboratory wavelength as indicated on the figure (small vertical line). After the flux equality of components, the intensity of the blueshifted one increases while the redshifted component decreases to disappearance. All metallic absorption lines that we have observed, from $370-570 \mathrm{~nm}$, reveal the line doubling phenomenon except the neutral ones which are affected by the disappearance phenomenon during the hump. The line doubling phenomenon takes place during $5 \%$ of the pulsation period, two times shorter than the observed one in RR Lyr (Chadid \& Gillet 1996a).

\section{Line emission phenomenon}

Both UVES and FEROS observations show that S Arae presents a strong blueshifted hydrogen emission with a smaller but significant redshifted one, associated with the hump, which is observed for $5 \%$ of the pulsation period centred on phase 0.95 . Figure 9 shows the strong hydrogen emission decreases from $\mathrm{H} \beta$ to $\mathrm{H} \delta$. The redshifted emission is clearly visible only in $\mathrm{H} \beta$.

Just after the hydrogen emission, the hydrogen profiles $\mathrm{H} \beta, \mathrm{H} \gamma$ and $\mathrm{H} \delta$ show a double absorption. Figure 8 presents the Balmer $\mathrm{H} \beta$ profile showing an emission followed by the doubling phenomenon and it is clear that the $\mathrm{H} \beta$ laboratory wavelength, which refers to the null velocity, is located between absorption components. The flux equality of the two line components occurs at phase 0.99 .

\section{Evidence of hypersonic shock waves in S Arae}

Zel'dovich \& Raiser (1966) discuss the schematic fine shock wave structure. They subdivided the shock wave into several principal zones with different physical characteristics: the precursor zone, the shock front, the thermalization zone, the

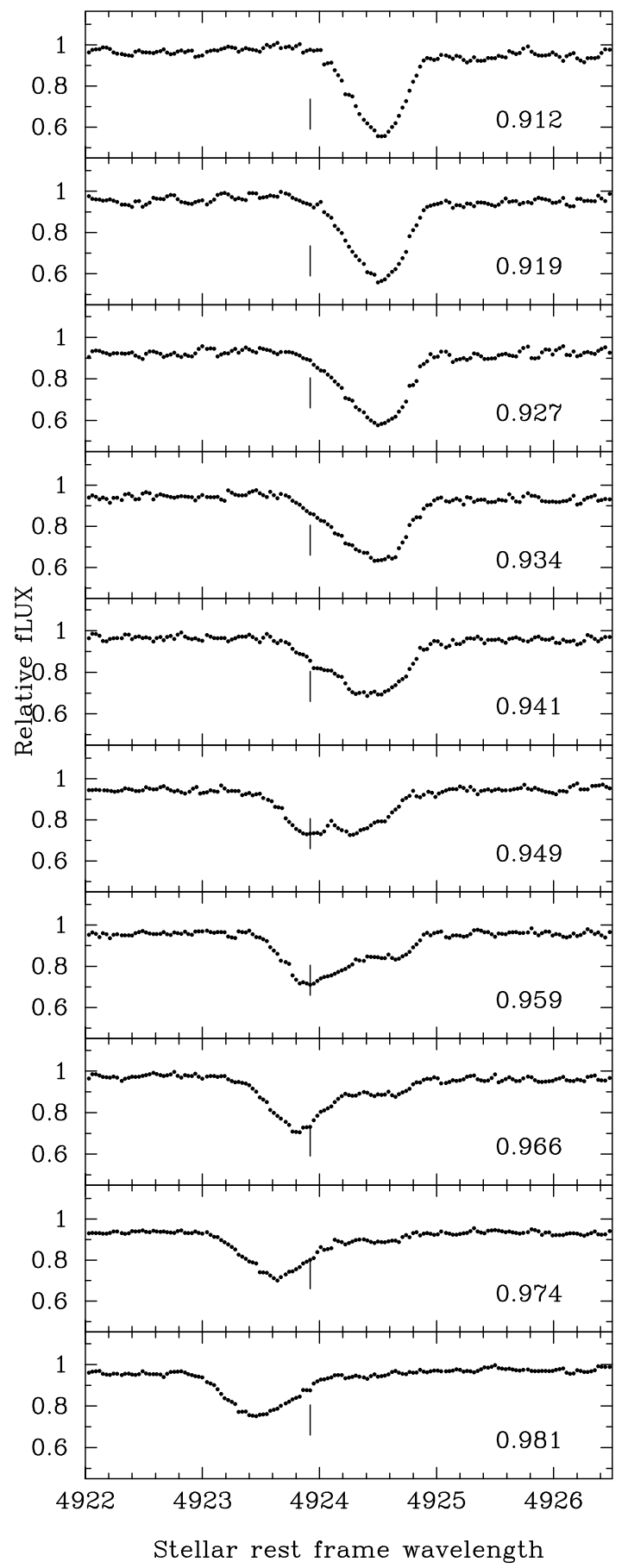

Fig. 7. UVES observed profiles of the single ionized metallic absorption line $\lambda \lambda 4923.921 \mathrm{Fe}$ II associated with the hump, showing a doubling. The phase is labeled in the lower right corner; the small vertical line gives the position of the laboratory wavelength.

ionization zone and the zone of the cooling wake. For the observer, this latter is the most important zone, because it is directly connected to the formation of the observed emission lines and their interpretation. It is partially transparent both in the Balmer and higher continua and in weak spectral lines. The whole shock structure is very sensitive to the degree of ionization in the precursor, especially if it was initially weak.

As has been mentioned by Anderson (2006), at high Mach numbers $(\geq 10)$, the shock is fully dominated by radiative phenomena. When the flowfield velocity becomes progressively 


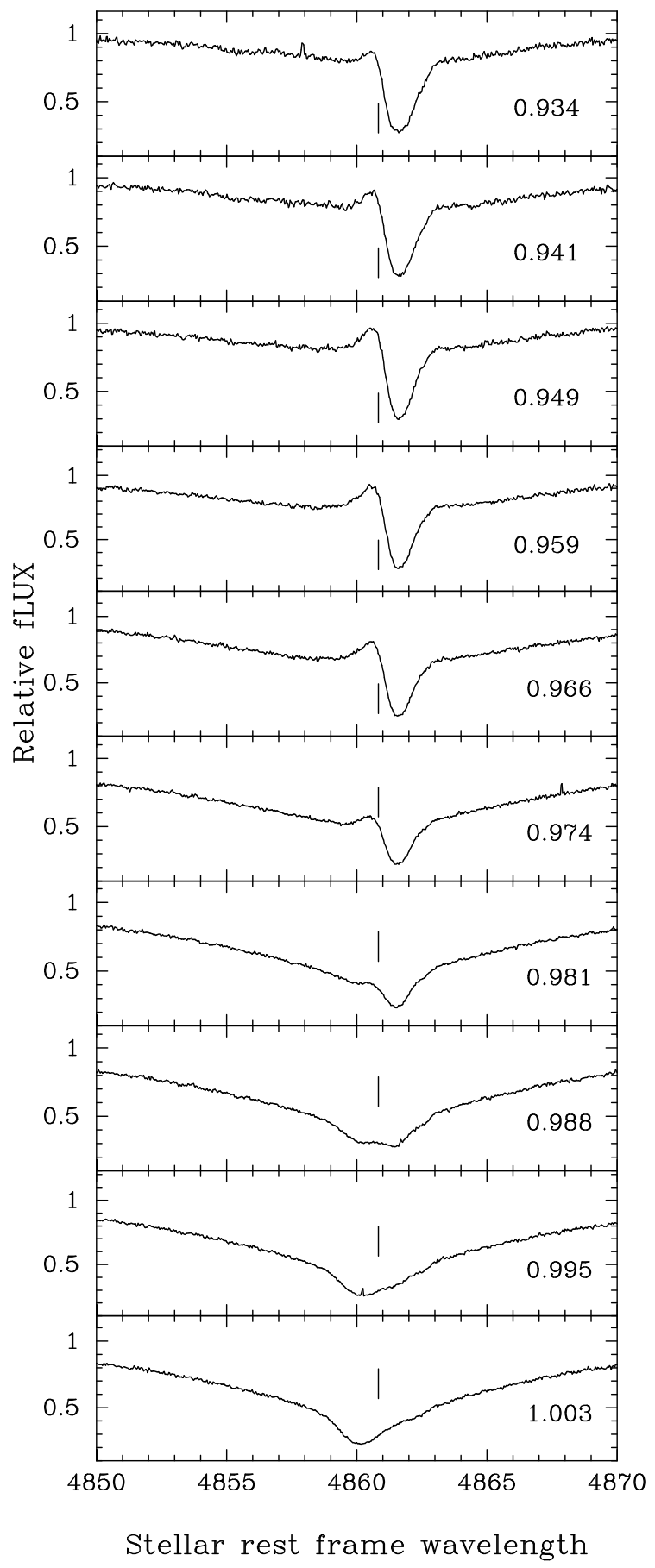

Fig. 8. UVES observed profiles of the $\mathrm{H} \beta$ hydrogen lines, associated with the hump, showing an emission followed by the doubling. The phase is labeled in the lower right corner; the small vertical lines give the position of the line laboratory wavelength.

higher, the temperature becomes high enough to generate the excitation, the dissociation and the ionization of molecules and atoms. Consequently, a fluid element radiates a substantial amount of energy. In such a flow, the fluid element loses energy due to the emission of radiation, but it can also gain energy due to the absorption of the emitted radiation from other fluid elements. The flowfield becomes nonadiabatic, i.e. its total enthalpy becomes variable and the radiative flux must be appreciable with respect to other hydrodynamical and thermodynamical fluid variables. In hypersonic shock waves, the coupling between hydrodynamic and radiative fields is high and can completely dominate the shock structure through the transfer equation, which is nonlocal. Hereafter, in this paper, we define the hypersonic shock as a shock wave where radiative phenomena have a major impact.

As shown in Sect. 4, during the crossing of the main shock, connected with the peak of the primary acceleration, all neutral metallic absorption lines exhibit a short line disappearance within an interval of around $8 \%$ of the pulsation period. During this time interval, all neutral metallic elements are single ionized. We recall that the minimum energy required to ionize a neutral atom of $\mathrm{Fe}, \mathrm{Ti}, \mathrm{Mg}$ and $\mathrm{Ca}$ to the first ion is respectively $7.87,6.82,7.64$ and $6.11 \mathrm{eV}$, equivalent to the ionization threshold temperatures between $70921 \mathrm{~K}$ and $91334 \mathrm{~K}$.

The adiabatic Rankine-Hugoniot relationships, for a strong shock, give an estimation of the shock front velocity value:

$V_{\mathrm{s}}=\sqrt{\frac{16}{3} \frac{R}{\mu} T_{\mathrm{f}}}$

where $V_{\mathrm{s}}$ is in $\mathrm{km} \mathrm{s}^{-1}, \mu$ is the mean atomic weight, $R$ is the universal gas constant and $T_{\mathrm{f}}$ is the temperature just after the shock front when the heavy particles have recovered a new Maxwellian distribution. Note that $T_{\mathrm{f}}$ applies to the gas before all ionizations take place and consequently, it is higher than the temperature of the ionization (Liberman \& Velikovich 1986). Thus, one can assume that $T_{\mathrm{f}}$ is very high and goes at least to $91334 \mathrm{~K}$. From formula (4), the shock front velocity value $V_{\mathrm{s}}$ is higher than $70 \mathrm{~km} \mathrm{~s}^{-1}$ which is about 10 times larger than the sound velocity (shock Mach number $\sim 10$ ). Thus, just above the S Arae photosphere, there is hypersonic motion enabling the ionization from atoms to ions.

The temporal evolution of the doubling phenomenon is shown to be in general agreement with the Schwarzschild mechanism (1952). In S Arae, since the extension of the ballistic photospheric motion is considerable, we interpret the line doubling of the single ionized metallic lines (Fig. 7) as a consequence of the two-step Schwarzchild mechanism (Chadid \& Gillet 1996a). A new shock wave crosses the deepest atmospheric layers when the ballistic motion induced by the previous shock is far from being relaxed. The shock at the begining drifts with the atmosphere, crossing the line forming region, its velocity is at first less than the infalling material and consequently the shock is at first a receding shock at phase 0.912. After fitting the red-shifted broad line profile with two Gaussians, it appears that the component is made of two redshifted lines centered at $+30.30 \mathrm{~km} \mathrm{~s}^{-1}$ and $+48.68 \mathrm{~km} \mathrm{~s}^{-1}$. Thus, the shock amplitude at the level of metallic absorption lines, which roughly corresponds to the separation between components of the double absorption line, is around $25 \mathrm{~km} \mathrm{~s}^{-1}$. This value is relatively weak in producing a double phenomenon, and consequently only a broadening line is observed first. Then the shock is strongly accelerated during its propagation above the photosphere and, afterwards, a stationary shock appears around phase 0.941 , when one of the two of components is close to zero velocity. We detect a maximum separation between the blueshifted and redshifted components of $0.98 \AA$. This means that the shock amplitude is around $81 \mathrm{~km} \mathrm{~s}^{-1}$. The advancing shock reaches the highest layers of the region where the Fe II line forms at phases 0.949-0.966 and the redshifted component disappears more and more. Finally, the shock leaves the Fe II region completely at phase 0.981 . At phase 0.974 , the blueshifted velocity component of the residual Fe II absorption line increases as the consequence of the expansion of the atmosphere (see Figs. 3 and 4). 


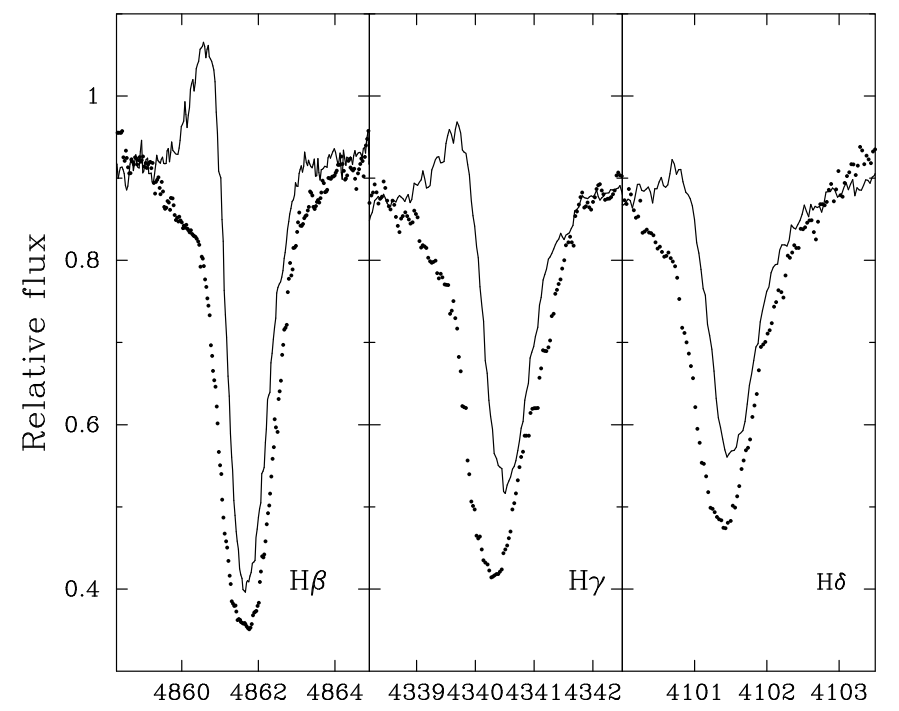

Stellar rest frame wavelength

Fig. 9. UVES hydrogen lines at the maximum observed emission $\phi=$ 0.95 (solid line) compared to those at phase $\phi=0.85$ (filled circles). The double emission structure is only visible in the $\mathrm{H} \beta$ profile. The profile at phase 0.85 is only in absorption.

The shock, which is propagating in a rapidly decreasing density region, becomes stronger and stronger during its propagation in the high atmosphere where the core of hydrogen line forms. The maximum difference between the redshifted and blueshifted hydrogen profile showing doubling (Fig. 8) is equal to $2.58 \AA$ and consequentely, the shock amplitude observed in the outermost atmospheric layers of $S$ Arae is around $217 \mathrm{~km} \mathrm{~s}^{-1}$.

As a result, we must infer that the shock amplitude where the core of metallic absorption lines forms is at least equal to $81 \mathrm{~km} \mathrm{~s}^{-1}$, which is about 12 times the sound velocity (shock Mach number $\sim 12$ ). This amplitude increases up to a maximun value in the highest part of the atmosphere where the hydrogen line core forms. It is at least about 33 times the sound velocity (shock Mach number $~ 33$ ). Such extremely high velocities imply that the shock is fully radiative and there is hypersonic motion in the whole atmosphere, from the photosphere to the high atmosphere of S Arae.

The $\mathrm{H} \beta$ profile shows the double emission structure associated with the hump (Fig. 9). A comparison with the $\mathrm{H} \beta$ profile (fitted circles) without emission shows double emission components. The blueshifted contribution is stronger than the redshifted one. This means that the double emission is apparent and is the consequence of a combination of a single large emission and a strong photospheric absorption. This is consistent with an absorption dip, smaller at phase 0.95 than at phase 0.85 . According to Gillet (1988) the double emission structure observed in the Mira Balmer emission is a consequence of a high shock front velocity. Up to now, only the $\mathrm{H} \alpha$ double emission structure was suspected to be detected in RR Lyrae stars and, in particular, in X Ari (Gillet \& Crowe 1988). This means that the shock front velocity in S Arae is higher than the one in X Ari since if the emission is weak, only a blue-shifted emission component is visible as in the cas of $\mathrm{H} \gamma$ and $\mathrm{H} \delta$ (Fig. 9). Finally, the presence of this double emission within the $\mathrm{H} \beta$ profile is consistent with hypersonic shock waves.

\section{Conclusion}

Using new high resolution spectroscopic observations obtained during one pulsation period of large amplitude RR Lyrae star S Arae, for the first time: (1) a new observed mechanism - line disappearance - detected in the neutral metallic absorption lines around the luminosity maximum and during a phase interval of $8 \%$ of the pulsation period; (2) the line doubling phenomenon is two times shorter than the observed one in RR Lyr (5\% of the pulsation cycle); (3) the rising time is very short, corresponding to $\sim 10 \%$, and the intensity of the pulsating motion and the extension of the atmosphere in S Arae is two times greater than those in RR Lyr; and (4) the $\mathrm{H} \beta$ double emission structure is clearly detected.

We have interpreted the observed results as a consequence of a hypersonic shock wave propagating through the photosphere towards the high atmosphere of S Arae. The hypersonic shock velocity is almost three times greater in the high atmosphere than the deep one. Our new results imply that renewed investigation of other nonlinear, nonadiabatic pulsational models, taking into account the presence of hypersonic shock waves in the pulsating atmosphere, must be undertaken.

Acknowledgements. It is a real pleasure to acknowledge the referee G. Bono for the very positive comments and suggestions. M. Chadid thanks C. Lidman and A. Kaufer for fruitful discussions.

\section{References}

Anderson, J. 2006, Hypersonic and High-Temperature Gas Dynamics Second Edition, AIAA Education Series

Bono, G., Caputo, F., \& Stellingwerf, R. F. 1994, ApJ, 432, L51

Bono, G., Caputo, F., castellani, V., \& Marconi, M. 1997, A\&A, 121, 327

Blazhko, S. 1907, Astron Nachr., 175, 325

Bowen, G. H. 1988, ApJ, 329, 299

Breitfellner, M. G., \& Gillet, D. 1993, A\&A, 277, 553

Burki, G., Meylan, M., \& Benz, W. 1982, A\&A, 109, 267

Chadid, M. 2000, A\&A, 359, 991

Chadid, M., \& Gillet, D. 1996a, A\&A, 308, 481

Chadid, M., \& Gillet, D. 1996b, A\&A, 315, 475

Chadid, M., Gillet, D., \& Fokin, A. 2000, A\&A, 363, 568

Fokin, A., Massacrier, G., \& Gillet, D. 2000 A\&A, 355, 668

Gillet, D. 1988, A\&A, 192, 206

Gillet, D., \& Crowe, R. A. 1988, A\&A, 199, 242

Gillet, D., Burki, G., \& Crowe, R. A. 1989, A\&A, 225, 455

Hill, S. J. 1972, ApJ, 178, 793

Iroshnikov, R. S. 1962, SvA, 5, 475

Jones, R. V., Carney B. W., Latham, D. W., \& Kurucz, R. L. 1987, ApJ, 312, 254

Kaufer, A., D’Odorico, S., \& Kaper, L. 2004, UVES User Manual, Issue 1.9, VLT-MAN-ESO-13200-1825

Kurucz, R. L. 1993, CD-ROM 23, 13, 18

Liberman, M. A., \& Velikovich, A. L. 1986, Physics of Shock Waves in Gases and Plasmas (Berlin, Heidelberg, New York: Springer), 43

Oke, J. B. 1966, ApJ, 145, 468

Perryman, M. A. C. 1997, A\&A, 323, 49

Preston, G. W. 1961, ApJ, 134, 633

Pritchard, J. D. 2004, FEROS-II User Manual, Issue 1.4, LSO-MAN- ESO22200-0001

Sanford, R. 1949, ApJ, 109, 208

Schwarzschild, M. 1952, in Transactions of the IAU VIII, ed. P. T. Oosterhoff (Cambridge: Cambridge Univ. Press), 811

Wils, P., \& Sodor, A. 2005, IBVS, 5655

Willson, L. A., \& Hill, S. J. 1979, ApJ, 228, 854

Zel'dovich, Ya. B., \& Raizer, Yu. P. 1966, Physics of shock waves and high temperature hydrodynamic phenomena (New-York: Academic Press) 
M. Chadid et al.: Hypersonic shock wave evidence in RR Lyrae stars, Online Material $p 1$

Table 1. Journal of UVES spectroscopic observations of S Arae with the Very Large Telescope Kuyen (VLT-UT2).

\begin{tabular}{lcc}
\hline \hline Date & HJD & Pulsation phase \\
\hline & $2400000+$ & \\
\hline 2001 Jul. 23 & 52113.5242654 & 0.822 \\
2001 Jul. 23 & 52113.5275638 & 0.829 \\
2001 Jul. 23 & 52113.5308623 & 0.836 \\
2001 Jul. 23 & 52113.5341376 & 0.843 \\
2001 Jul. 23 & 52113.5374361 & 0.851 \\
2001 Jul. 23 & 52113.5407229 & 0.858 \\
2001 Jul. 23 & 52113.5440214 & 0.865 \\
2001 Jul. 23 & 52113.5473314 & 0.873 \\
2001 Jul. 23 & 52113.5506299 & 0.880 \\
2001 Jul. 23 & 52113.5552709 & 0.890 \\
2001 Jul. 23 & 52113.5585578 & 0.897 \\
2001 Jul. 23 & 52113.5651547 & 0.912 \\
2001 Jul. 23 & 52113.5684763 & 0.919 \\
2001 Jul. 23 & 52113.5717632 & 0.927 \\
2001 Jul. 23 & 52113.5750733 & 0.934 \\
2001 Jul. 23 & 52113.5783717 & 0.941 \\
2001 Jul. 23 & 52113.5816586 & 0.949 \\
2001 Jul. 23 & 52113.5863228 & 0.959 \\
2001 Jul. 23 & 52113.5896212 & 0.966 \\
2001 Jul. 23 & 52113.5929313 & 0.974 \\
2001 Jul. 23 & 52113.5962181 & 0.981 \\
2001 Jul. 23 & 52113.5995166 & 0.988 \\
2001 Jul. 23 & 52113.6028151 & 0.995 \\
2001 Jul. 23 & 52113.6061020 & 1.002 \\
2001 Jul. 23 & 52113.6094120 & 1.010 \\
2001 Jul. 23 & 52113.6126989 & 1.017 \\
2001 Jul. 23 & 52113.6173746 & 1.028 \\
2001 Jul. 23 & 52113.6206731 & 1.035 \\
2001 Jul. 23 & 52113.6239599 & 1.042 \\
2001 Jul. 23 & 52113.6272468 & 1.049 \\
2001 Jul. 23 & 52113.6305569 & 1.057 \\
2001 Jul. 23 & 52113.6338669 & 1.064 \\
2001 Jul. 23 & 52113.6371654 & 1.071 \\
2001 Jul. 23 & 52113.6404754 & 1.079 \\
2001 Jul. 23 & 52113.6437739 & 1.086 \\
\hline & & \\
& & \\
\hline
\end{tabular}

Table 2. Journal of FEROS spectroscopic observations of S Arae with the $1.50 \mathrm{~m}$ telescope.

\begin{tabular}{|c|c|c|}
\hline Date & $\begin{array}{c}\text { HJD } \\
2400000+\end{array}$ & Pulsation phase \\
\hline 2001 Jun. 29 & 52090.4567307 & 0.774 \\
\hline 2001 Jun. 29 & 52090.4661749 & 0.795 \\
\hline 2001 Jun. 29 & 52090.4759895 & 0.817 \\
\hline 2001 Jun. 29 & 52090.4887786 & 0.845 \\
\hline 2001 Jun. 29 & 52090.5018917 & 0.874 \\
\hline 2001 Jun. 29 & 52090.5149007 & 0.903 \\
\hline 2001 Jun. 29 & 52090.5381409 & 0.954 \\
\hline 2001 Jun. 29 & 52090.5615663 & 1.006 \\
\hline 2001 Jun. 29 & 52090.5847255 & 1.057 \\
\hline 2001 Jun. 29 & 52090.6083246 & 1.109 \\
\hline 2001 Jun. 29 & 52090.6444233 & 1.189 \\
\hline 2001 Jun. 29 & 52090.6572124 & 1.218 \\
\hline 2001 Jun. 29 & 52090.6681381 & 1.242 \\
\hline 2001 Jun. 29 & 52090.6987855 & 1.310 \\
\hline 2001 Jun. 29 & 52090.7094913 & 1.333 \\
\hline 2001 Jun. 29 & 52090.7228012 & 1.363 \\
\hline 2001 Jun. 29 & 52090.7359259 & 1.392 \\
\hline 2001 Jun. 29 & 52090.7516779 & 1.427 \\
\hline 2001 Jun. 29 & 52090.7648026 & 1.456 \\
\hline 2001 Jun. 29 & 52090.7780199 & 1.485 \\
\hline 2001 Jun. 29 & 52090.7910983 & 1.514 \\
\hline 2001 Jun. 29 & 52090.8084591 & 1.552 \\
\hline 2001 Jun. 29 & 52090.8316414 & 1.604 \\
\hline 2001 Jun. 29 & 52090.8505415 & 1.645 \\
\hline 2001 Jun. 29 & 52090.8668027 & 1.681 \\
\hline 2001 Jun. 29 & 52090.8831102 & 1.718 \\
\hline 2001 Jun. 29 & 52090.9001585 & 1.755 \\
\hline
\end{tabular}

